\title{
Determination of Prominence Plasma $\beta$ from the Dynamics of Rising Plumes
}

\author{
Andrew Hillier ${ }^{1}$, Richard Hillier ${ }^{2}$ and Durgesh Tripathi ${ }^{3}$ \\ ${ }^{1}$ Kwasan Observatory, 17-1 Kitakazan-ohmine-cho, Yamashina-ku, Kyoto, 607-8471, Japan \\ email: andrew@kwasan.kyoto-u.ac.jp \\ ${ }^{2}$ Department of Aeronautics, Imperial College, London, United Kingdom \\ ${ }^{3}$ Inter-University Centre for Astronomy and Astrophysics, Post Bag 4, Ganeshkhind, Pune 411 \\ 007, India
}

\begin{abstract}
Observations of quiescent prominences show rising plumes, dark in chromospheric lines, that propagate from large bubbles. In this paper we present a method that may be used to determine the plasma $\beta$ (ratio of gas pressure to magnetic pressure) from the rising plumes. Using the classic fluid dynamic solution for flow around a circular cylinder, the compression of the prominence material can be estimated. Application to a prominence gave an estimate of the plasma $\beta$ as $\beta=0.47-1.13$ for a ratio of specific heats of $\gamma=1.4-1.7$.
\end{abstract}

Keywords. Sun: prominences, magnetic fields, MHD, instabilities

\section{Introduction}

Understanding the strength of the magnetic field of prominences is of great importance for the study of prominence dynamics and space weather forecasting. Measurements of the magnetic field for quiet sun prominences and filaments gives a range of 3 to $30 \mathrm{G}$ (e.g. Leroy 1989; Casini et al. 2009; Schmieder et al. 2013). However, the sample of prominences that have had their magnetic field strength measured is still very small, meaning that other methods are often employed to estimate the strength of a prominence's magnetic field. Commonly used methods include prominence seismology (e.g. Arregui et al. 2012; Ballester 2013) and extrapolation of the photospheric magnetic field (e.g. Aulanier \& Démoulin 2003; van Ballegooijen 2013).

Since the launch of the Hinode satellite, there has been great focus on the dynamics of the rising plumes (see Figure 1) (Berger et al. 2008; de Toma et al. 2008; Berger et al. 2010) created by the magnetic Rayleigh-Taylor instability (Berger et al. 2010; Hillier et al. 2011, 2012a). As any instability, wave or flow that is observed in an Magnetohydrodynamic (MHD) system should contain information about the system itself, these rising plumes present a great opportunity to investigate the plasma parameters of quiescent prominences. In this paper, we present a method that uses observations by the Solar Optical Telescope (SOT; Tsuneta et al. 2008) on the Hinode satellite (Kosugi et al. 2007) of rising plumes to determine the plasma $\beta$ of a quiescent prominence. A full explanation of the methodology and results can be found in Hillier et al. (2012b)

\section{Necessary Information to Determine Prominence Plasma $\beta$}

The method to determine the plasma $\beta$ of a prominence, as presented in (Hillier et al. $2012 \mathrm{~b}$ ), is formulated using a set of observational information about prominences and their dynamics. Before presenting the methodology, first it is necessary to quickly review 
these observational characteristics of prominences, and in particular their plumes, that are important for this analysis. The points we need to remember are:

- The plumes rise at an approximately constant velocity (Berger et al. 2010)

- The plumes have an elliptical head (Berger et al. 2010)

- The intensity of the prominence material at the top of the plume is greater than the average prominence intensity (Hillier et al. $2012 \mathrm{~b}$ )

- Flows of material can be observed along the head of the plume (Berger et al. 2011)

- The prominence magnetic field is mainly horizontal (e.g. Schmieder et al. 2013)

First we must relate the prominence intensity to the density of the prominence. The prominence intensity is dominated by scattering, giving the intensity as proportional to the column density. Hence the bright top of the plume would imply that there has been an increase in the density through compression of the prominence.

Next we can use the approximately constant velocity and elliptical plume head to perform two coordinate transforms that greatly simplify the situation under consideration. First we remove the plume stem, treating it as a rising ellipse in the prominence material. As this ellipse is moving at constant velocity through (what we assume to be) a constant medium, a shift in reference frame gives a stationary ellipse in a constant flow. Now we must consider the 3D nature of the geometry under investigation. The observed plumes are known to be caused by the magnetic Rayleigh-Taylor instability (Berger et al. 2010; Hillier et al. 2012a), which creates filamentary structures aligned with the magnetic field (Isobe et al. 2005, 2006b; Stone \& Gardiner 2007; Hillier et al. 2012a) (for no shear in the magnetic field). Therefore, we can view the problem as being similar of an elliptical cylinder in a constant flow. Performing a conformal transform of the coordinate system to change to a circular cylinder creates a situation analogous to that of a constant flow around a circular cylinder.

For simplicity, we will now make the assumption that the plasma $\beta$ is approximately uniform. Based on this assumption, and modifying the derivation for a compressible flow around a circular cylinder (van Dyke 1975), we find:

$$
\begin{aligned}
& \rho=\left(1-\frac{\gamma-1}{2} M_{*}^{2}\left(v_{r}^{2}+v_{\theta}^{2}-1\right)\right)^{1 /(\gamma-1)} \\
& v_{r}(r, \theta)=\left(1-\frac{1}{r^{2}}\right) \cos \theta \\
&-M_{*}^{2}\left[\left(-\frac{13}{12} \frac{1}{r^{2}}+\frac{3}{2} \frac{1}{r^{4}}-\frac{5}{12} \frac{1}{r^{6}}\right) \cos \theta+\left(\frac{1}{4} \frac{1}{r^{4}}-\frac{1}{4} \frac{1}{r^{2}}\right) \cos ^{3} \theta\right]+O\left(M_{*}^{4}\right) \\
& v_{\theta}(r, \theta)=-\left(1+\frac{1}{r^{2}}\right) \sin \theta \\
&+M_{*}^{2}\left[\left(\frac{13}{12} \frac{1}{r^{2}}-\frac{1}{2} \frac{1}{r^{4}}+\frac{1}{12} \frac{1}{r^{6}}\right) \sin \theta-\left(\frac{1}{12} \frac{1}{r^{4}}-\frac{1}{4} \frac{1}{r^{2}}\right) 3 \cos ^{2} \theta \sin \theta\right]+O\left(M_{*}^{4}\right) \\
& M_{*}= \sqrt{\frac{\gamma \beta_{\infty}}{\gamma \beta_{\infty}+2(\gamma-1)}} M_{\infty}
\end{aligned}
$$

Equation 2.1 can now be used to investigate the compression around a plume head.

\section{Application to a Prominence Plume}

In this section we apply the method presented above to determine the plasma $\beta$ of the plume displayed in the panel on the right of Figure 1. Due to the short timescales of 

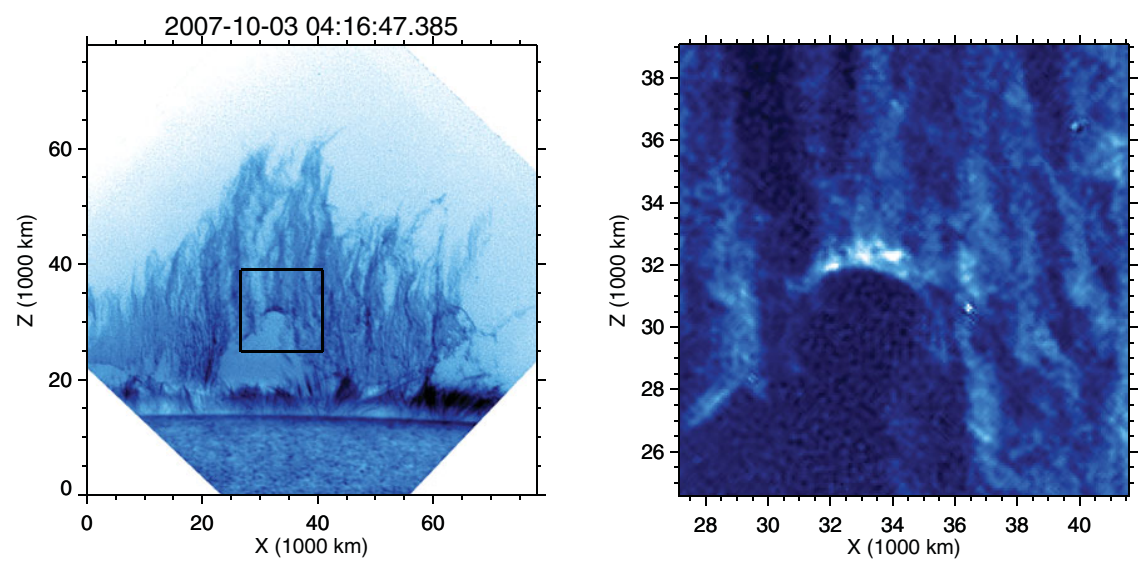

Figure 1. The left-hand panel shows a quiescent prominence observed on the 3-Oct-2007 at 04:16UT. The right-hand panel shows a zoom-in of the region in the box in the left-hand panel. The plume and the bright prominence plasma above it are clearly visible.

these compressions $(\sim 100 \mathrm{~s})$, we assume that the increased intensity comes through an increased column density resulting from compression.

Using the plume observations shown in Figure 1, it is possible to determine the Mach number of the rising plume and the plume dimensions. This prominence was observed on the NW solar limb on 2007-10-03 observed by the SOT with the Ca II H filter at a cadence of $30 \mathrm{~s}$. The velocity of the plume was found to be approximately constant, with velocity and the velocity error calculated to be $v_{\text {plume }}=12.3 \pm 0.6 \mathrm{~km} \mathrm{~s}^{-1}$. The sound speed of the prominence material is $C_{s}=\left[\gamma(R / \mu) T_{\text {prom }}\right]^{1 / 2}=11 \mathrm{~km} \mathrm{~s}^{-1}$ for $\gamma=5 / 3$, $\mu=0.9$ and $T_{\text {prom }}=8000 \mathrm{~K}$ giving a Mach number of the flow of $M_{\infty}=1.12 \pm 0.05$. The dimensions of the elliptical plume head are $1.3 \operatorname{arcsec}$ and $2.35 \operatorname{arcsec}$ for the short and long axes, these are used for the conformal transform of the plume head.

Figure 2 shows the normalised intensity, which is used as a proxy for the column density. The fit is shown by the dotted line. The fitting error is determined as the standard deviation of the difference between the normalised intensity and the fit at each pixel and the intensity error is determined by the intensity fluctuations of the stray light in the corona. The error bars equivalent to $2 \hat{\sigma}_{\text {prom }}$ and $2 \hat{\sigma}_{f i t}$ are shown in Figure 2.

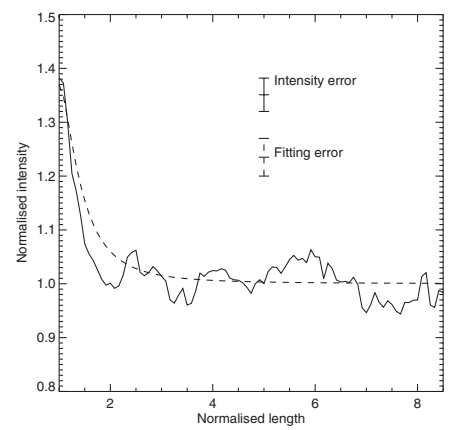

Figure 2. The graph shows a plot of intensity along the slit (solid line) and the fitting assuming the intensity proportional to density (dashed line) for $\gamma=1.65$. Error bars equivalent to twice the standard deviation are presented for the observed intensity and the fit. 
Using the value of $M_{*}$ from the fitting of the intensity and the definition of $M_{*}$ as given in Equation 2.4, it is possible to solve for the plasma $\beta$ of the prominence, where $\beta$ is given by:

$$
\beta=\frac{2(\gamma-1)}{\gamma} \frac{M_{*}^{2}}{M_{\infty}^{2}-M_{*}^{2}}
$$

Assuming the range $\gamma=1.4-1.7$, we find a plasma $\beta$ of $\beta=0.47 \pm 0.079$ to $1.13 \pm 0.080$.

\section{Conclusion}

In this paper, we presented a method to determine a prominence's plasma $\beta$. Applying Equation 2.1 to observations of a prominence we found that for the range $\gamma=1.4-1.7$ the observed prominence has a plasma $\beta$ of $\beta=0.47 \pm 0.079$ to $1.13 \pm 0.080$. This method can be applied to any plume observation to provide an estimate of the plasma $\beta$ of the prominence in which the plumes are formed.

One interesting point to note is that we have equations that define the velocity around the plume head. Therefore, through analysis of the velocities at the observed plume head, the projection of the plume onto the plane-of-sky can be determined. Using this information, an estimation of the direction of the magnetic field in the prominence may be possible.

Hinode is a Japanese mission developed and launched by ISAS/JAXA, with NAOJ as domestic partner and NASA and STFC (UK) as international partners. It is operated by these agencies in co-operation with ESA and NSC (Norway). AH is supported by KAKENHI Grant-in-Aid for Young Scientists (B) 25800108.

\section{References}

Arregui, I., Oliver, R., \& Ballester, J. L. 2012, Living Reviews in Solar Physics, 9, 2

Aulanier, G. \& Démoulin, P. 2003, A \& A, 402, 769

Ballester, J. L. 2013, The IAU Symposium 300 Proceedings 2013, in press

Berger, T. E., et al. 2008, ApJ Letters, 676, L89

Berger, T. E., et al. 2010, ApJ, 716, 1288

Berger, T., et al. 2011, Nature, 472, 197

Casini, R., López Ariste, A., Paletou, F., \& Léger, L. 2009, ApJ, 703, 114

Chae, J. 2010, ApJ, 714, 618

de Toma, G., Casini, R., Burkepile, J. T., \& Low, B. C. 2008, ApJ Letters, 687, L123

Hillier, A., Isobe, H., Shibata, K., \& Berger, T. 2011b, ApJ Letters, 736, L1

Hillier, A., Berger, T., Isobe, H., \& Shibata, K. 2012a, ApJ, 746, 120

Hillier, A., Hillier, R., \& Tripathi, D. 2012b, ApJ, 761, 106

Isobe, H., Miyagoshi, T., Shibata, K., \& Yokoyama, T. 2005, Nature, 434, 478

Isobe, H., Miyagoshi, T., Shibata, K., \& Yokoyama, T. 2006b, PASJ, 58, 423

Kosugi, T., et al. 2007, Sol. Phys., 243, 3

Leroy, J. L. 1989, Dynamics and Structure of Quiescent Solar Prominences, 150, 77

López Ariste, A. 2013, The IAU Symposium 300 Proceedings 2013, in press

Schmieder, B., Kucera, T. A., Knizhnik, K., et al. 2013, arXiv:1309.1568

Stone, J. M. \& Gardiner, T. 2007, ApJ, 671, 1726

Tsuneta, S., et al. 2008, Sol. Phys., 249, 167

van Ballegooijen, A. 2013, The IAU Symposium 300 Proceedings 2013, in press

van Dyke, M. 1975, NASA STI/Recon Technical Report A, 75, 46926 